\title{
Mortalidad por desnutrición en menores de cinco años, Colombia, 2003-2007
}

\author{
Edwin Fernando Quiroga \\ Subdirección de Vigilancia y Control en Salud Pública, Instituto Nacional de Salud, Bogotá D.C., Colombia \\ Entidad donde realiza el estudio: Universidad Externado de Colombia
}

Introducción. Los mayores problemas de mortalidad en menores de cinco años se presentan en países con altos índices de pobreza, lo que demuestra la iniquidad en relación con los países desarrollados. Se han identificado causas generales de mortalidad, como trastornos perinatales, sarampión, VIH/ sida, diarrea e infecciones respiratorias agudas, por lo que se hace énfasis en que la desnutrición ha tenido que ver con la mitad de ellas, aproximadamente, como una causa subyacente.

Objetivo. Caracterizar la mortalidad por desnutrición utilizando un método de ajuste para la tasa, dadas las dificultades en su estimación por las limitaciones en la identificación de la desnutrición como causa básica de muerte.

Materiales y métodos. Se llevó a cabo un estudio descriptivo que incluyó análisis de códigos CIE10 de las estadísticas vitales entre 2003-2007, con estimación de las tasas de mortalidad, aplicación del método de causas múltiples para enfermedades infecciosas y cálculo de las probabilidades de muerte.

Resultados. La desnutrición, como antecedente, tiene asociación con las enfermedades infecciosas; la frecuencia de la enfermedad infecciosa como causa básica de muerte fue siete veces mayor cuando coexistió la desnutrición como causa antecedente. Una vez ajustada la tasa de mortalidad, se encontró que aumentó cinco veces el valor inicial. El método de "análisis de causas múltiples" establece su efectividad como metodología en el ajuste de este tipo de mortalidad.

Conclusión. La desnutrición puede ser una causa básica o subyacente de muerte en una de cada ocho defunciones, en niños menores de un año, y en una de cada tres, en niños de uno a cuatro años.

Palabras clave: desnutrición proteica, Kwashiorkor, tasa de mortalidad, mortalidad infantil, clasificación internacional de enfermedades, estadísticas vitales.

doi: http://dx.doi.org/10.7705/biomedica.v32i4.741

\section{Death rate by malnutrition in children under the age of five, Colombia}

Introduction. Much higher mortalities occur in children under five in developing countries with high poverty rates compared with developed countries. Causes of death are related to perinatal conditions, measles, HIV/AIDS, diarrhea, respiratory diseases and others. Throughout the world, malnutrition has been identified as the underlying cause of approximately half of these deaths.

Objective. Death rate due to malnutrition was described using an adjusted method that takes into account the difficulties of identifying malnutrition as a direct cause of death.

Materials and methods. A descriptive study included analysis of the International Classification of Diseases (ICD-10) vital statistics from 2003-2007. Death rates were estimated, a method of analysis of multiple causes was applied for infectious diseases, along with calculations of death probabilities.

Results. Malnutrition was associated with infectious diseases. The frequency of infectious disease as a direct cause of death was almost seven times higher in cases with the antecedent of malnutrition. When adjusted death rate values were used, the initial value increased nearly five times. The probability of death after the adjustment for inadequate classification increased approximately four times. The Analysis of Multiple Causes Method was established as an effective method in analyzing malnutrition and infectious diesease mortality in Colombia.

Conclusion. Malnutrition may be a direct underlying cause of death in one of eight deaths in children $<1$ year old and one of three deaths in 1-4-year-olds.

Key words: Protein malnutrition, Kwashiorkor, mortality rate, infant mortality, international classification of diseases, vital statistics.

doi: http://dx.doi.org/10.7705/biomedica.v32i4.741 
En el mundo, $143^{\prime} 000.000$ de niños menores de cinco años presentan desnutrición (1). Se estima que es causa fundamental de $53 \%$ de todas las muertes de niños menores de cinco años $(1,2)$. Estas muertes representan solamente una parte de los niños que sufren de desnutrición en los países en desarrollo; sin embargo, muchos de ellos sobreviven.

Para estos niños que sobreviven, la malnutrición a edades tempranas genera un incremento en el riesgo de enfermedades infecciosas, baja talla, baja capacidad de aprendizaje y, a largo plazo, daño en el potencial de desarrollo físico, intelectual y productivo en la etapa adulta, con consecuencias para la familia, la comunidad, el país y el mundo (3), lo que repercute finalmente en una alta carga económica y limitación del desarrollo humano. La desnutrición es el resultado de múltiples factores asociados: económicos, políticos y sociales, y es, a la vez, la causante de múltiples problemas de salud, como la vulnerabilidad ante las infecciones recurrentes, que contribuyen a las altas tasas de mortalidad en esta población (4). Se ha identificado una serie de causas generales de mortalidad en menores de cinco años, entre las que se destacan los trastornos perinatales, el $\mathrm{VIH} / \mathrm{sida}$, la diarrea, las infecciones respiratorias agudas y otras, por lo que es importante hacer énfasis en que la desnutrición ha tenido que ver con la mitad de ellas, aproximadamente, como una causa subyacente de una de cada dos muertes (5).

La importancia y necesidad de definir la desnutrición como causa de mortalidad, radican en aspectos mencionados por organismos internacionales, los cuales especifican que "La desnutrición severa (sic.) mata 10'800.000 niños menores de cinco años en el mundo cada año"(6). De la misma manera, la Organización Mundial de la Salud (OMS) define la desnutrición como un "rango de condiciones fisiopatológicas, que se presentan como consecuencia de la deficiencia conjunta de proteínas y calorías en diferentes proporciones, que se presenta más frecuentemente en lactantes y que se asocia más comúnmente a infecciones" $(7,8)$. A partir de lo anterior, es preciso tener una aproximación de esta condición dentro de la

Correspondencia:

Edwin Fernando Quiroga, Diagonal 82G N 78-88, Bogotá, D.C., Colombia

Teléfonos: (571) 2233424 y (300) 2679633

ferquirogav@gmail.com

Recibido: 05/10/11; aceptado:16/05/12 mortalidad infantil que se registra como alguna causa asociada o antecedente.

Asimismo, la Food and Agriculture Organization (FAO) refiere que la interacción o sinergismo de la malnutrición y la infección, es la causa principal de morbilidad y mortalidad de los niños en la mayoría de los países de África, Asia y América Latina. Las infecciones de tipo viral, bacteriano y parasitarias tienden a ser prevalentes, y todas pueden tener un impacto negativo en el estado nutricional, especialmente, en menores de cinco años y adultos mayores. Esta relación sinérgica entre la malnutrición y las enfermedades infecciosas, es el resultado de una interacción que tiene consecuencias más serias sobre los niños de lo que tendría el efecto aditivo si las dos se presentaran de modo independiente. Las infecciones empeoran la malnutrición y ésta aumenta la gravedad de las enfermedades infecciosas, llevándolas al círculo conocido como desnutrición-infección (9).

Según la reciente publicación de la Encuesta Nacional de la Situación Nutricional (ENSIN, 2010), hay una reducción en las prevalencias de desnutrición aguda y global en los niños menores de cinco años (10), que son las que se encuentran directamente relacionadas con la mortalidad. Esta encuesta compara sus resultados con los publicados desde 1990 por la encuesta nacional de demografía y salud en sus ediciones quinquenales hasta el año 2010 (11), e informa que la desnutrición global en este grupo de edad pasó de 8,6 \%, en 1990 , a $3,4 \%$, en el 2010 , y que la desnutrición aguda disminuyó de $1,4 \%$, en 1995 , a $0,7 \%$, en el 2010 (10).

En el 2004 se llevó a cabo en Colombia una investigación sobre la mortalidad asociada a la desnutrición. Según este estudio, entre 1998 y 2002, en el país ocurrieron 39.197 defunciones en las que estuvo presente la desnutrición ya fuera como causa directa, como causa antecedente o como otro estado patológico importante en el momento de la defunción; además, demostró que en los niños menores de cinco años, existe la probabilidad de que una de cada cinco muertes sea por desnutrición o esté asociada a esta, como sucede en la mayor parte de los municipios del país (5).

En Colombia, para los menores de cinco años las causas de muerte debidas a procesos infecciosos, principalmente respiratorios y del aparato gastrointestinal, son los que se ubican como causa básica en los certificados de defunción, en 
muchos de los cuales interviene la malnutrición, lo cual ocasiona una inadecuada clasificación de las infecciones como causa de mortalidad (12). Esta situación es atribuible, en alguna medida, a la falta de reconocimiento y definición de la desnutrición como hecho patológico que puede llevar a la muerte (13).

De esta manera, como resultado de las reglas de codificación de la Clasificación Internacional de Enfermedades (14), se propone como causa básica a una que no fue la que originó la cadena de eventos que llevaron a la muerte. Por lo tanto, mediante el estudio de las causas múltiples, puede conocerse la influencia de ciertas causas en esta cadena de eventos.

Lo anteriormente expuesto, sobre los problemas en la clasificación que puede existir en la mortalidad infantil y de la niñez cuando la causa básica pudo haber sido la desnutrición, evidencia la necesidad de estudiar esta condición relacionándola con las causas infecciosas, para permitir una aproximación al verdadero valor que debe tener la tasa de mortalidad infantil (menores de un año) y de la niñez (de uno a cuatro años) por esta causa (cuadro 1).

El objetivo de este trabajo fue describir el comportamiento de la mortalidad por desnutrición en menores de cinco años, a partir de la serie de tiempo 2003 a 2007 para Colombia, evaluando la fuente de información, estimando las tasas y probabilidades de muerte por esta condición y ajustando estas medidas por el método de causas múltiples, con el fin de contar con una mejor aproximación a esta problemática.

\section{Materiales y métodos}

En esta investigación de tipo descriptivo, se caracterizó la mortalidad por desnutrición cuando es una causa básica y antecedente, en comparación con las enfermedades infecciosas. La unidad de análisis correspondió a las defunciones según causas de muerte en menores de cinco años, correspondientes a los códigos CIE-10, registrados por el sistema de estadísticas vitales, administrado por el DANE (15), para Colombia entre el 2003 y 2007. Se utilizó para el análisis el total de las defunciones en niños menores de cinco años, por lo cual no se requirió el cálculo de muestra. Para el caso de los menores de un año, y dada la significancia que amerita este grupo de edad y la trascendencia de la medida por su comportamiento diferencial en comparación con otros grupos de edad, se analizó por separado del grupo de uno a cuatro años; así, se estimaron la la tasa de mortalidad infantil (menores de un año) por 100.000 nacidos vivos y la tasa de mortalidad en la niñez con la población de uno a cuatro años $(5,11)$.

Se utilizaron las variables del certificado de defunción, tales como año de la defunción, edad del fallecido, departamento de residencia y causas de muerte según los códigos CIE-10.

Se utilizaron tres fuentes de información complementarias para la obtención de las tasas y medidas de asociación (cuadro 1): 1) defunciones por grupos de edad y sexo para el país, según lista de causas para el año 2003 a 2007; 2) nacimientos a nivel nacional, para los años 2003 a 2007, y 3) censo 2005 y proyecciones de población.

Las causas asociadas a la mortalidad por desnutrición, que se describen ampliamente en la literatura científica, sonlas de origen infeccioso (enfermedades respiratorias agudas y enfermedades diarreicas agudas), las cuales según la CIE-10 corresponden a los códigos entre A000 y B990, enfermedades infecciosas, entre J000 y J220, infecciones de origen respiratorio y $\mathrm{P} 783$, diarrea neonatal no infecciosa (14), las cuales se utilizaron para hacer el análisis de causas múltiples.

Cuadro 1. Estimación de tasas de mortalidad

Tasa de mortalidad infantil $=$ Defunciones en niños $<$ de 1 año por desnutrición (año X) x 1.000

por desnutrición

Total de nacidos vivos (año $\mathrm{X}$ )

Tasa de mortalidad en la niñez $=$ Defunciones en niños de 1 a 4 años por desnutrición (año X) x 1.000

por desnutrición

Total de niños de 1 a 4 años (año X)

La tasa de mortalidad infantil relaciona las defunciones de menores de un año acaecidas durante un año y el número de nacidos vivos registrados en el transcurso del mismo año. Se considera como uno de los indicadores más importantes para la planificación en salud y, debido a su especial utilidad, es necesario calcular e interpretar correctamente sus valores.

Fuente: United Nations. Manual X: Indirect Techniques for Demographic Estimation. United Nations Publication, Sales No. E.83. XIII.2, 1983. 
A partir de la causa básica de defunción (de origen infeccioso) se determinó cuáles de ellas estuvieron asociadas a la desnutrición, así como las causas antecedentes que permitieron hacer el análisis de causas múltiples.

El método de análisis de causas múltiples consiste en la tabulación de las causas básicas y causas antecedentes registradas (en este caso, del certificado de defunción, las causas b, c, d y otros estados patológicos importantes). Una vez identificadas las causas básicas (las enfermedades infecciosas), se llevó a cabo la estimación de medidas de asociación entre las causas antecedentes (en el numerador) y las causas básicas (en el denominador), denominándola cociente debido a su aplicación más universal (cuadro 2). Se utilizó la medida de asociación razón de disparidad, OR (odds ratio), que busca determinar en qué medida las causas múltiples están asociadas con las básicas (16); se utilizó para establecer la asociación (no causal), en este caso, entre la desnutrición y las causas de muerte de origen infeccioso, a fin de comparar entre los estados patológicos adyacentes o condicionantes cuando el registrado como definitivo o de deceso es otro, que resultó como consecuencia de los primeros (15).

Para la estimación de las probabilidades de muerte por desnutrición, según el método de Greville, (cuadro 3), se tomaron los nacimientos y las defunciones para todos los años del período, estas últimas divididas en los factores de separación, para calcular los sobrevivientes a la edad más un año $(x+1)$ y las personas menores de un año al primero de enero de cada año, así como las defunciones de uno a cuatro años; estos registros se tomaron sin ajustar (17).

A partir del diagrama de Lexis (18), tomando los nacimientos en cada año y las defunciones por desnutrición, se estimaron las probabilidades de muerte para los menores de un año y los niños de uno a cuatro años. Una vez obtenidas las probabilidades de supervivencia en cada uno de los grupos, se calculó la probabilidad de muerte conjunta para el grupo de cero a cinco años para el total nacional. Para el cálculo de las probabilidades de muerte por el método de Greville, se separaron las defunciones de menores de un año de edad y de uno a cuatro años, lo que permite, además, estimar las tasas de mortalidad infantil (defunciones en menores de un año respecto de los nacidos vivos en el mismo periodo) y de mortalidad de la niñez (mortalidad en niños de uno a cuatro años respecto a la población de la misma edad) (17).

Las bases de datos se encuentran originalmente en formato de base de datos, las cuales se trabajaron en Access $^{\circledast}$ y Excel $^{\circledR}$ de Microsoft Office. El plan de análisis consistió inicialmente en generar las proporciones de omisiones y faltantes de información, así como la generación de tasas y probabilidades de muerte, las cuales se trabajaron en este último software y se utilizó de manera complementaria Epilnfo ${ }^{\mathrm{TM}}$, versión 5.3.2, el cual es de licencia gratuita. Para la estimación de razones de disparidad, se empleó el software EpiDat ${ }^{\mathrm{TM}}$, versión 3.1, también de distribución gratuita.

\section{Consideraciones éticas}

Se tuvo en cuenta la Resolución 8430 de 1993, donde prevalece el respeto a la dignidad y protección de los derechos y el bienestar de los sujetos de investigación. Según la Ley 23 de 1981, Código de Ética Médica, se ajustaron los principios metodológicos y éticos para salvaguardar los derechos de las personas, manteniendo su integridad. La información utilizada no incluye datos de identidad de los menores fallecidos. En cuanto a la Declaración de Helsinki de la Asociación Médica Mundial, es un análisis descriptivo de la mortalidad, que pretende generar líneas de base para la toma de decisiones de política pública en salud.

Cuadro 2. Cálculo de medidas de asociación

\begin{tabular}{|c|c|c|c|c|}
\hline \multirow{2}{*}{ Cálculo de la razón de disparidad (OR) } & & Presente & Ausente & Total \\
\hline & Expuesto & a & $b$ & $a+b$ \\
\hline$b \times c$ & No expuesto & $c$ & $d$ & $c+d$ \\
\hline & Total & $a+c$ & $b+d$ & $a+b+c+d$ \\
\hline
\end{tabular}

a.Menor de 5 años - causa básica infecciosa donde la desnutrición fue una causa antecedente.

b.Menor de 5 años - causa básica diferente a infecciosa donde la desnutrición fue una causa antecedente.

c. Menor de 5 años - causa básica infecciosa donde la desnutrición no fue una causa antecedente.

d. Menor de 5 años - causa básica diferente a infecciosa donde la desnutrición no fue una causa antecedente 
Cuadro 3. Cálculo de probabilidades con el método de Greville El cálculo de las probabilidades de muerte por el método Greville, se realiza con diagrama de Lexis a partir de la identificación de las defunciones y factores de separación, teniendo en cuenta edades exactas y cumplidas, de acuerdo con las siguientes formulas:

$E x=E_{x}^{z-1}+E_{x}^{z}+E_{x}^{z+1}$

$\mathrm{N}^{\prime \prime} \mathrm{x}=\mathrm{N}_{\mathrm{x}}^{\mathrm{z}-1}+\mathrm{N}_{\mathrm{x}}^{\mathrm{z}}+\mathrm{N}_{\mathrm{x}}^{\mathrm{z}+1}$

$N^{\prime} x=N_{x}^{z}+N_{x}^{z+1}+N_{x}^{z+2}$

Ex $=$ número de niños a la edad exacta de 0 (nacimientos), 1 , 2, 3 y 4 años

$\mathrm{Nx}=$ número de niños con edad cumplida al año $\mathrm{x}$

$\mathrm{Z}=$ año de referencia

El método consiste en hallar las probabilidades de supervivencia y, a partir de esta medida (el complemento 1-P), permite establecer la $q_{0}$ y la ${ }_{4} q_{1}$. Para hallar la probabilidad de muerte en menores de 5 años, es decir ${ }_{4} q_{0}$, como probabilidad conjunta, se hace a partir del producto de las probabilidades de supervivencia de las dos anteriores.

$$
\begin{aligned}
& { }_{4} p_{0}=p_{0} \times{ }_{4} p_{1} \\
& { }_{4} q_{0}=1-{ }_{4} p_{0}
\end{aligned}
$$

\section{Resultados}

\section{Calidad de la fuente de información}

De las 180.000 defunciones que se presentan en promedio cada año en Colombia, el $7 \%$ corresponde a menores de cinco años. Se revisaron 69.964 registros de defunción (en bases de datos) en el periodo de estudio, cerca de 14.000 por año. La mayoría de las variables (excepto el sexo) presentó omisiones de contenido que impidieron su adecuado análisis para la caracterización de la mortalidad por desnutrición en Colombia, en el periodo 2003-2007 (cuadro 4).

La información que se clasificó como faltante corresponde a los campos vacíos, es decir, sin información; lo que se puede observar para las variables presentadas anteriormente es que la omisión del sexo es de $0 \%$ en todos los años.
La calidad de esta variable es adecuada y provee la información para el análisis. Sin embargo, las variables de afiliación al Sistema de Seguridad Social en Salud (SGSSS), presenta porcentajes de omisión que sobrepasan el $10 \%$ (entre 1.466 y 3.278 registros), lo cual hace que la calidad de esta información sea deficiente.

Lo mismo sucede con variables que podrían estar relacionadas directamente con la mortalidad por desnutrición, como el bajo peso al nacer. Sin embargo, no es posible utilizar dicha variable dado que presenta porcentajes de omisión que van desde $17 \%$, para el 2007 , hasta $49 \%$, en el 2003 (faltantes hasta de 7.474 registros). Aunque la edad de la madre y el nivel educativo podrían aportar información valiosa en los diferenciales de la mortalidad infantil y de la niñez, en este estudio no fue práctico hacer el ajuste con porcentajes tan altos de faltantes, de $55 \%$ (8.301 registros) en el 2003 y de $40 \%$ (5.258 registros) en el 2007).

Las variables que hacen referencia a las causas, el año de la defunción y el sexo, son las que presentan una mejor calidad, con porcentajes de omisión o faltantes inferiores al $5 \%$ en el periodo de estudio.

\section{Caracterización de la mortalidad por desnutrición en menores de cinco años de edad}

La mortalidad infantil por desnutrición se mantiene entre 5,6 y 6,7 por cada 100.000 nacidos vivos para el periodo de referencia, teniendo en cuenta que en promedio se registran al año 720.000 nacimientos en el país; para este grupo de edad no se observa una reducción año a año, pues la tasa se reduce en el año 2005 y aumenta nuevamente en el 2006 y el 2007.

La tasa de mortalidad en la niñez sí muestra una reducción año a año y se encuentra entre 0,55 y

\begin{tabular}{|c|c|c|c|c|c|c|c|c|c|c|}
\hline \multirow[b]{3}{*}{ Año } & \multicolumn{10}{|c|}{$\begin{array}{l}\text { Número y porcentaje de faltantes (en blanco o sin información), } \\
\text { de las variablesde interés en la mortalidad en menores de cinco años }\end{array}$} \\
\hline & \multicolumn{2}{|c|}{$\begin{array}{l}\text { Sexo del } \\
\text { fallecido }\end{array}$} & \multicolumn{2}{|c|}{$\begin{array}{l}\text { Seguridad } \\
\text { social }\end{array}$} & \multicolumn{2}{|c|}{$\begin{array}{l}\text { Peso al nacer } \\
\text { del fallecido }\end{array}$} & \multicolumn{2}{|c|}{$\begin{array}{l}\text { Edad de la madre } \\
\text { del fallecido }\end{array}$} & \multicolumn{2}{|c|}{$\begin{array}{l}\text { Nivel educativo } \\
\text { de la madre }\end{array}$} \\
\hline & $\mathbf{n}$ & $\%$ & $\mathrm{n}$ & $\%$ & $\mathbf{n}$ & $\%$ & $\mathbf{n}$ & $\%$ & $n$ & $\%$ \\
\hline 2003 & 0 & 0 & 3.278 & 21,8 & 7.474 & 49,7 & 7.173 & 47,7 & 8.301 & 55,2 \\
\hline 2004 & 0 & 0 & 2.636 & 18,3 & 5.113 & 35,5 & 5.013 & 34,8 & 6.251 & 43,4 \\
\hline 2005 & 0 & 0 & 2.193 & 15,7 & 3.142 & 22,5 & 3.142 & 22,5 & 6.550 & 46,9 \\
\hline 2006 & 0 & 0 & 1.466 & 10,9 & 4.733 & 35,2 & 4.693 & 34,9 & 5.728 & 42,6 \\
\hline 2007 & 0 & 0 & 1.704 & 13,0 & 2.281 & 17,4 & 2.006 & 15,3 & 5.258 & 40,1 \\
\hline
\end{tabular}

Cuadro 4. Completitud de las variables de interés para las defunciones en menores de cinco años, Colombia, 2003-2007 
Cuadro 5. Tasas de mortalidad por desnutrición para el 2003 a 2007

\begin{tabular}{lcc}
\hline Año & \multicolumn{2}{c}{ *Tasas de mortalidad por desnutrición en menores de cinco años } \\
\cline { 2 - 3 } & $\begin{array}{c}\text { Mortalidad infantil } \\
\text { (por cada 100.000 nacidos vivos) }\end{array}$ & $\begin{array}{c}\text { Mortalidad niñez } \\
\text { (por cada 100.000 habitantes entre 1 y 4 años) }\end{array}$ \\
\hline 2003 & 6,7 & 1,21 \\
2004 & 6,4 & 0,86 \\
2005 & 5,6 & 0,83 \\
2006 & 5,9 & 0,67 \\
2007 & 6,1 & 0.55 \\
\hline
\end{tabular}

* La estimación de las tasas se hizo sin ajustar por subregistro.

Cuadro 6. Tasas de mortalidad por desnutrición para el 2003 a 2007, según el sexo.

\begin{tabular}{ccccc}
\hline Año & \multicolumn{3}{c}{ *Tasas de mortalidad por desnutrición en menores de cinco años según el sexo } \\
\cline { 2 - 5 } & \multicolumn{2}{c}{$\begin{array}{c}\text { Mortalidad infantil } \\
\text { (por cada 100.000 nacidos vivos) } \\
\text { Masculino }\end{array}$} & $\begin{array}{c}\text { Mortalidad niñez } \\
\text { Femenino }\end{array}$ & $\begin{array}{c}\text { (por cada 100.000 habitantes entre 1 y 4 años) } \\
\text { Masculino }\end{array}$ \\
\hline 2003 & 6,59 & 6,88 & 1,11 & 1,32 \\
2004 & 6,52 & 6,37 & 0,95 & 0,76 \\
2005 & 5,14 & 6,05 & 0,97 & 0,68 \\
2006 & 5,53 & 6,31 & 0,58 & 0,79 \\
2007 & 5,87 & 6,49 & 0,45 & 0,65 \\
\hline
\end{tabular}

* La estimación de las tasas se hizo sin ajustar por subregistro.

1,21 por cada 100.000 menores entre uno y cuatro años de edad, para el periodo estudiado (cuadro 5).

Se observó que no hay diferencias de la desnutrición como causa básica de defunción comparada según el sexo; es decir, esta variable no se asume como un diferencial para la mortalidad por desnutrición (cuadro 6).

Desnutrición como causa antecedente en las enfermedades infecciosas

Los decesos de origen infeccioso para la causa básica de muerte fueron 1.478, aproximadamente el $10 \%$ del total de mortalidad en menores de cinco años; de estos, se registró la desnutrición como causa antecedente, en promedio para el periodo, en 236 fallecimientos; es decir, en promedio en el $16 \%$ de los fallecimientos debido a causas infecciosas, la desnutrición estuvo presente como una causa antecedente de defunción (cuadro 7).

A partir de estos datos y teniendo en cuenta el total de defunciones en menores de cinco años, el resultado de la OR (cuadro 8 ) en promedio fue de 7,11 para el periodo 2003 a 2007 (cuadro 9), lo cual indica que la mortalidad en menores de cinco años debida a desnutrición como causa antecedente, efectivamente se relaciona con las enfermedades infecciosas como causa básica de muerte.

Los intervalos de confianza permiten confirmar dicha asociación. La frecuencia de la enfermedad

Cuadro 7. Número de defunciones por causas infecciosas asociadas a la desnutrición y número de causas en las cuales estuvo presente la desnutrición como causa antecedente de muerte

\begin{tabular}{lcc}
\hline Año & $\begin{array}{c}\text { Número de defunciones por causas asociadas a la desnutrición en menores } \\
\text { de cinco años y causa antecedente de desnutrición }\end{array}$ \\
\cline { 2 - 3 } & $\begin{array}{c}\text { Causa básica } \\
\text { enfermedades infecciosas }\end{array}$ & $\begin{array}{c}\text { Causa antecedente } \\
\text { de desnutrición }\end{array}$ \\
\hline 2003 & 1.648 & 310 \\
2004 & 1.570 & 249 \\
2005 & 1.461 & 241 \\
2006 & 1.434 & 203 \\
2007 & 1.277 & 178 \\
Total & 7.390 & 1.181 \\
\hline
\end{tabular}


Cuadro 8. Estimaciones de las razones de disparidad en menores de cinco años de edad, entre el 2003 y 2007

\begin{tabular}{|c|c|c|c|c|c|c|c|c|}
\hline AÑO 2003 & & & & AÑO & 2004 & & & \\
\hline $\begin{array}{l}\text { Defunciones por } \\
\text { desnutrición (causas } \\
\text { antecedentes } 1+2+3 \text { ) }\end{array}$ & $\begin{array}{l}\text { Defuncion } \\
\text { por (enferr } \\
\text { infecciosas }\end{array}$ & $\begin{array}{l}\text { ¿5 años } \\
\text { es }\end{array}$ & Total & $\begin{array}{l}\text { Defun } \\
\text { desnu } \\
\text { antece }\end{array}$ & $\begin{array}{l}\text { es por } \\
\text { ón (causas } \\
\text { tes } 1+2+3)\end{array}$ & $\begin{array}{l}\text { Defuncio } \\
\text { por (enfe } \\
\text { infeccios }\end{array}$ & $\begin{array}{l}\text { an }<5 \text { años } \\
\text { dades }\end{array}$ & Total \\
\hline & $\begin{array}{l}\text { Sí } \\
\text { (cuando fu } \\
\text { por causa }\end{array}$ & No & & & & $\begin{array}{r}\text { Sí } \\
\text { (cuando } \\
\text { por cause }\end{array}$ & No & \\
\hline Sí & 310 & 395 & 705 & Sí & & 249 & 337 & 586 \\
\hline No & 1.379 & 12.954 & 14.333 & No & & 1.321 & 12.497 & 13.818 \\
\hline Total & 1.689 & 13.349 & 15.038 & Total & & 1.570 & 12.834 & 14.404 \\
\hline $\mathrm{OR}=7,37$ & & & & $\mathrm{OR}=$ & & & & \\
\hline AÑO 2005 & & & & AÑO & 2006 & & & \\
\hline $\begin{array}{l}\text { Defunciones por } \\
\text { desnutrición (causas } \\
\text { antecedentes } 1+2+3 \text { ) }\end{array}$ & $\begin{array}{l}\text { Defuncion } \\
\text { por (enferr } \\
\text { infecciosas }\end{array}$ & $\begin{array}{l}\text { < } 5 \text { años } \\
\text { es }\end{array}$ & Total & $\begin{array}{l}\text { Defun } \\
\text { desnu } \\
\text { antece }\end{array}$ & $\begin{array}{l}\text { es por } \\
\text { ón (causas } \\
\text { tes } 1+2+3)\end{array}$ & $\begin{array}{l}\text { Defuncio } \\
\text { por (enfe } \\
\text { infeccios }\end{array}$ & $\begin{array}{l}\text { en años } \\
\text { dades }\end{array}$ & Total \\
\hline & $\begin{array}{l}\text { Sí } \\
\text { (cuando fu } \\
\text { por causa }\end{array}$ & No & & & & $\begin{array}{c}\text { Sí } \\
\text { (cuando } \\
\text { por caus }\end{array}$ & No & \\
\hline & 241 & 325 & 566 & & & 203 & 304 & 507 \\
\hline & 1.220 & 12.179 & 13.399 & & & 1.231 & 11.708 & 12.939 \\
\hline & 1.461 & 12.504 & 13.965 & & & 1.434 & 12.012 & 13.446 \\
\hline $\mathrm{OR}=7,40$ & & & & & & & & \\
\hline AÑO 2007 & & & & & & & & \\
\hline $\begin{array}{l}\text { Defunciones por } \\
\text { desnutrición (causas } \\
\text { antecedentes } 1+2+3 \text { ) }\end{array}$ & $\begin{array}{l}\text { Defuncion } \\
\text { por (enfer } \\
\text { infecciosas }\end{array}$ & $\begin{array}{l}5 \text { años } \\
\text { les }\end{array}$ & Total & & & & & \\
\hline & $\begin{array}{c}\text { Sí } \\
\text { (cuando f } \\
\text { por causa }\end{array}$ & No & & & & & & \\
\hline Sí & 178 & 252 & 430 & & & & & \\
\hline No & 1.099 & 11.582 & 12.681 & & & & & \\
\hline Total & 1.277 & 11.834 & 13.111 & & & & & \\
\hline
\end{tabular}

$\mathrm{OR}=7,44$

Cuadro 9. Resumen de medidas de asociación, defunciones por causas infecciosas asociadas a la desnutrición en menores de cinco años

\begin{tabular}{lc}
\hline Año & $\begin{array}{c}\text { Medidas de asociación } \\
\text { OR }\end{array}$ \\
\hline 2003 & 7,37 \\
2004 & 6,99 \\
2005 & 7,40 \\
2006 & 6,35 \\
2007 & 7,44 \\
Promedio & 7,11 \\
\hline
\end{tabular}

infecciosa como causa básica de muerte fue siete veces mayor cuando coexistió la desnutrición como causa antecedente.

Este resultado permite evidenciar que la desnutrición, como una causa básica de muerte en este grupo de población, efectivamente presenta problemas de clasificación, dado que se puede considerar una causa incorrecta, en este caso las enfermedades de origen infeccioso como la infección respiratoria aguda o la diarreica aguda, cuando probablemente la desnutrición pudo ser la causa básica de defunción.

\section{Propuesta de ajuste de las tasas de mortalidad por desnutrición}

Al ajustar las causas de defunción por desnutrición, a partir de los resultados del método de causas múltiples, se encontró que el número de defunciones por desnutrición aumenta más de cuatro veces, lo cual permite plantear que la desnutrición podría estar mal clasificada cuando es una causa básica de muerte (cuadro 10).

Para hacer el ajuste de las defunciones por desnutrición como causa básica, se tabularon uno a uno los registros de la base de datos, en los cuales la causa básica fue una enfermedad infecciosa que 
Cuadro 10. Ajuste de las defunciones por desnutrición, a partir del método de causas múltiples

\begin{tabular}{ccc}
\hline Año & \multicolumn{2}{c}{$\begin{array}{c}\text { Número de defunciones por desnutrición } \\
\text { en menores de cinco años ajustadas, } \\
\text { después del análisis de causas múltiples }\end{array}$} \\
\cline { 2 - 3 } & $\begin{array}{c}\text { Causa } \\
\text { sin ajustar }\end{array}$ & $\begin{array}{c}\text { Causa } \\
\text { ajustada }\end{array}$ \\
\hline 2003 & 77 & 387 \\
2004 & 76 & 325 \\
2005 & 66 & 307 \\
2006 & 62 & 265 \\
2007 & 62 & 240 \\
Total & 343 & 1.524 \\
\hline
\end{tabular}

tuvo como causa antecedente (antecedente 1, 20 3) la desnutrición.

Al calcular las tasas de mortalidad ajustadas, se encontró que los valores aumentan alrededor de cinco veces el valor inicial; así, la tasa de mortalidad infantil se estima, en promedio, en 27 por cada 100.000 nacidos vivos para el periodo 2003 a 2007, y en 3,13 por cada 100.000 niños, en la tasa de mortalidad en la niñez para el mismo periodo (cuadro 11).

Estimación de las probabilidades de muerte por desnutrición en menores de cinco años, según el método de Greville

Las probabilidades de muerte por desnutrición aumentaron cuatro veces al ajustar el número de defunciones con el método de causas múltiples. Al comparar las probabilidades de muerte por desnutrición ajustadas, en relación con la probabilidad de muerte nacional para el periodo 2005-2010 (menores de un año: 0,01978; uno a cuatro años: 0,00470) (19), se observó que la probabilidad de muerte por desnutrición en menores de un año corresponde al $12,6 \%$ de la nacional y al $31,9 \%$ para la probabilidad de muerte en el grupo de uno a cuatro años. Esto refleja la carga o el peso de la mortalidad por desnutrición en el grupo de menores de cinco años. Puede decirse que la desnutrición es una causa básica o subyacente de muerte en una de cada ocho defunciones en niños menores de un año y, en una de cada tres, en niños de uno a cuatro años (cuadro 12).

\section{Discusión}

Al evaluar la fuente de información, en términos de calidad de los datos mediante el análisis de contenido, se determinó que las variables sociodemográficas que podrían estar relacionadas con el estudio de la mortalidad por desnutrición en menores de cinco años, presentan dificultades importantes relacionadas con los datos faltantes que impiden caracterizar los factores de riesgo 0 determinantes de la mortalidad por desnutrición en este grupo de edad. Este análisis refleja la calidad de la fuente de información desde el punto de vista de variables complementarias del sistema de estadísticas vitales. Sin embargo, es importante mencionar que año a año la omisión de contenido

Cuadro 11. Ajuste de tasas de mortalidad por desnutrición en menores de cinco años, a partir del método de causas múltiples

\begin{tabular}{ccc}
\hline Año & $\begin{array}{c}\text { Tasas de mortalidad por desnutrición en menores de cinco años } \\
\text { ajustadas según el método de causas múltiples }\end{array}$ \\
\cline { 2 - 3 } & $\begin{array}{c}\text { Mortalidad infantil } \\
\text { (por cada 100.000 } \\
\text { nacidos vivos) }\end{array}$ & $\begin{array}{c}\text { Mortalidad niñez } \\
\text { (por cada 100.000 habitantes } \\
\text { entre 1 } \mathbf{y} \mathbf{4} \text { años) }\end{array}$ \\
\hline 2003 & 32,4 & 4,33 \\
2004 & 28,3 & 3,42 \\
2005 & 27,9 & 3,03 \\
2006 & 24,6 & 2,63 \\
2007 & 22,8 & 2,26 \\
\hline
\end{tabular}

Cuadro 12. Probabilidades de muerte por desnutrición en menores de cinco años, método Greville.

\begin{tabular}{lcc}
\hline $\begin{array}{l}\text { Probabilidad de muerte } \\
\text { por grupo de edad }\end{array}$ & $\begin{array}{c}\text { Probabilidades de muerte por desnutrición en menores de cinco años } \\
\text { ajustadas según el método de causas múltiples }\end{array}$ \\
\cline { 2 - 3 } & Sin ajustar & Ajustada \\
\hline $\mathrm{q}_{0}$ & 0,000706 & 0,002563 \\
${ }_{4} \mathrm{q}_{1}$ & 0,00033 & 0,00159 \\
${ }_{4} \mathrm{q}_{0}$ & 0,00104 & 0,00415 \\
\hline
\end{tabular}


de las variables disminuye significativamente, lo cual permite suponer que en el corto plazo la omisión puede ser inferior al 10 \% y, así, mejorar la calidad del registro.

La mortalidad por desnutrición como causa antecedente tiene una asociación no causal con las enfermedades infecciosas como causas básicas de muerte; como se mencionó anteriormente, aunque no es posible establecer si la desnutrición fue causa antecedente o efecto en el episodio que finalmente produjo la muerte, sí se puede afirmar que es el resultado del círculo o interacción infección-desnutrición-infección (9). Así, el presente estudio permitió establecer que la frecuencia de la enfermedad infecciosa como causa básica de muerte fue siete veces mayor cuando coexistió la desnutrición como causa antecedente, para el quinquenio delestudio. Alcomparar estos resultados con los presentados por Bustamante y Romero, en su publicación "El análisis de la desnutrición como causa múltiple de muerte" (16), se observa que, cuando no se consideró la desnutrición entre los acontecimientos del fallecimiento, la infección apareció casi ocho veces más frecuentemente como causa básica de muerte, que cuando coexistió la desnutrición como causa múltiple. Lo anterior permite hacer una aproximación a la relevancia de la interacción entre desnutrición e infección como binomio patológico de consecuencias extremas, en particular, en la población infantil. Definitivamente, según la estructura que actualmente tienen los certificados de defunción en Colombia (11), el estudio de causas múltiples permitió aproximarse a la inadecuada clasificación que puede generar en la tasa de mortalidad por desnutrición infantil y de la niñez, con el fin de proponer su ajuste y evidenciar la necesidad de intervenirla por su carácter de evitable.

Las tasas de mortalidad ajustadas aumentaron alrededor de cinco veces, cifras que van en aumento al compararlas con las estimaciones del estudio llevado a cabo entre 1998 y 2002, lo cual pone de manifiesto la importancia que puede tener esta alteración en los decesos totales de los menores de 5 años (5).

El cálculo de las probabilidades de muerte por desnutrición en menores de cinco años, utilizando el método de Greville, permite aproximarse al nivel de mortalidad por esta condición, la cual aumentó más de cuatro veces cuando se ajustaron las defunciones con el método de causas múltiples. Estas probabilidades no son comparables con el estudio del periodo 1998 a 2002, puesto que las probabilidades estimadas se presentan como tasas, mas no como una probabilidad que proviene de una cohorte hipotética como parte del estudio longitudinal (17).

Teniendo en cuenta lo anterior, en relación con los resultados del estudio de mortalidad por desnutrición en Colombia 1998-2002, donde concluyen que una de cada cinco defunciones en niños colombianos de uno a cuatro años de edad, está asociada con la desnutrición y en los menores de un año la proporción es de uno de cada diez defunciones (5), se puede observar que este tipo de mortalidad por desnutrición aumenta de manera importante en el grupo de edad estudiado.

En definitiva, la interacción entre desnutrición e infección afecta directamente la salud de los individuos, principalmente a aquellos grupos más vulnerables como niños y adultos mayores; sin embargo, la desnutrición generalmente no es considerada como una causa básica de muerte sino que constituye una causa condicionante o antecedente. Teniendo en cuanta lo anterior el estudio plantea que dentro de las causas múltiples, las antecedentes son aquellas que podrían tener menos sesgo por parte del médico, ya que su identificación no se relaciona con la cadena de eventos que en forma causal llevaron a la muerte.

En consecuencia a este planteamiento se propone que la frecuencia de mortalidad por causas antecedentes o asociadas puede ser una buena aproximación a la estimación de este tipo de mortalidad, clasificada como evitable, en la población.

Definitivamente el análisis de contenido de las variables puede ser una herramienta importante para la evaluación de la calidad de las diferentes fuentes de información, este ejercicio evidencia su utilidad como otra opción de evaluación para este tipo de registros. Para el estudio de este tipo de mortalidad, en especial por tratarse de menores de cinco años, la información faltante puede ser recuperada de otros registros, como el de nacidos vivos, para aproximarse más a los determinantes de la mortalidad por y asociada a la desnutrición en este grupo de población.

Los resultados de este estudio pueden aportar una línea de base en materia de política de primera infancia y al replanteamiento de las metas de la actual política pública de seguridad alimentaria y nutricional (20); además de servir como insumo en 
el seguimiento de las metas ya planteadas por el gobierno central y contribuir en la evaluación de cumplimiento de los objetivos de desarrollo del milenio, en términos de reducción de la mortalidad infantil.

Es importante profundizar, con otros estudios, los factores de riesgo que están presentes en este tipo de mortalidad con el fin de generar líneas de base que permitan plantear estrategias en el abordaje de la mortalidad por desnutrición como una mortalidad evitable.

Se recomienda hacer este mismo ejercicio a nivel departamental con el fin de evidenciar las diferencias que se presumen existen en territorios que tienen una mayor inseguridad alimentaria y nutricional y limitaciones en el acceso a los servicios de salud, y así establecer la magnitud de dicho problema y brindar información útil para la acción.

\section{Alcances y limitaciones del estudio}

El análisis de los registros de defunción permitió establecer a nivel nacional una aproximación del peso de la mortalidad asociada a la desnutrición en la mortalidad infantil y de la niñez, estimando las dificultades en la clasificación como causa básica de defunción por esta condición. Por los procedimientos del método y teniendo en cuenta los recursos disponibles incluyendo el tiempo, esta investigación sólo refleja el nivel de mortalidad a nivel nacional planteando un punto de partida para que en otras investigaciones se puedan hacer análisis similares en departamentos, distritos y municipios.

El método de causas múltiples permitió evidenciar la asociación (no causal) entre la desnutrición y enfermedades infecciosas como un modelo propuesto para el ajuste de la tasa de mortalidad por desnutrición; sin embargo, este método es una propuesta que debe tener una revisión detallada para su utilización, en caso de tomar los resultados como valores proximales para líneas de base o metas a nivel nacional.

Los modelos de ajustes de las tasas de mortalidad infantil no se pueden aplicar a una patología específica, por tanto las tasas no contienen este tipo de ajustes debido al subregistro.

\section{Agradecimientos}

Expreso mis agradecimientos a la Universidad Externado de Colombia y al Departamento Administrativo Nacional de Estadística. A Isabel Cristina Ruiz y Elizabeth Castellanos por sus aportes metodológicos y acompañamiento en el desarrollo de este estudio. A Máncel Martínez Ramos del Instituto Nacional de Salud, por sus aportes en la revisión y edición del documento para su publicación.

\section{Conflicto de intereses}

No existen conflictos de intereses

\section{Financiación}

Este proyecto se financió por parte del autor, como estudiante del programa de Especialización en Métodos de Análisis Demográfico y Maestría en Estudios de Población de la Universidad Externado de Colombia.

\section{Referencias}

1. Menchú MT, Santizo MC. Propuesta de indicadores para la vigilancia de la seguridad alimentaria y nutricional (SAN). Publicación INCAP PCE-073. Guatemala: INCAP/OPS/ OMS; 2002.

2. Puffer RR, Serrano CV. Patterns of mortality in childhood. Scientific Publication № 262. Washington, D.C.: Pan American Health Organization; 1973.

3. Fondo de las Naciones Unidas para la Infancia-UNICEF. Progreso para la infancia. Examen estadístico de un mundo apropiado para los niños y las niñas. 2007. Fecha de consulta: 2 de abril de 2011. Disponible en: http://www. unicef.org/spanish/publications/files/Progreso_para_la_ infancia_No_6.pdf]

4. Ortiz Mr, Álvarez-Dardet C, Ruiz M, Gascón E. Identificación de barreras a las políticas de nutrición y alimentación en Colombia: estudio por el método Delfos. Rev Panam Salud Pública. 2003;14:186-91. http://dx.doi. org/10.1590/S1020-49892003000800005.

5. Ruiz M, Ruiz N. La mortalidad por desnutrición en Colombia 1998-2002. Bogotá, D.C.; Editorial CIDS-Universidad Externado de Colombia; 2007.

6. Organización de las Naciones Unidas. Standing Committee on Nutrition. Takling de double burden of malnutrition. SCN News. 2006;32:1-76.

7. Organización Panamericana de la Salud. Vigilancia alimentaria y nutricional en las Américas. Publicación científica No.516. Washington, D.C.: OPS; 1989. p. 43.

8. Caballero B, O'Donnel Am, Torún S, Lara P, Bengoa J. Requerimientos nutricionales del niño enfermo en la alimentación del niño menor de seis años en América Latina. Bases para el desarrollo de guías de alimentación. Serie Paltex. Washington, D.C.: Organización Panamericana de la Salud; 1997. p. 1-10.

9. Organización para la Agricultura y la Alimentación FAO. Nutrición humana en el mundo en desarrollo. En: Nutrición e infección, salud y enfermedad. 2000. Fecha de consulta: 13 de abril de 2012. Disponible en: ftp://ftp.fao. org/docrep/fao/005/w0073s/W0073S00.pdf

10. Instituto Colombiano de Bienestar Familiar. Encuesta Nacional de la Situación Nutricional, ENSIN 2010. Primera edición. Bogotá, D.C.: Davinci Editores; 2011. 
11. Asociación Pro-Bienestar de la Familia ColombianaPROFAMILIA. Salud sexual y reproductiva: resultados de la Encuesta Nacional de Demografía y Salud, ENDS. Bogotá, D.C.: PROFAMILIA; 2005.

12. Organización Panamericana de la Salud. Programa de salud materno infantil y población. Fuentes de datos y defunciones utilizadas en salud materno infantil. Serie: HPM-CDR-SM 94-1E. Washington, D.C.: OPS; 1994.

13. Gómez R. La mortalidad evitable como indicador de desempeño de la política sanitaria en Colombia. 19952001. Revista Facultad Nacional de Salud Pública. 2006;1:3-54.

14. Organización Mundial de la Salud. Clasificación central de la WHO. Family of International Classifications (WHOFIC). International Statistical Classification of Diseases and Related Health Problems.Segunda edición. Washington, D.C.: OMS; 2004.

15. Departamento Administrativo de Estadística Nacional. Ficha metodológica -EEVV. Octubre de 2009. Fecha de consulta: 23 de febrero de 2011. Disponible en: http://www. dane.gov.co/files/investigaciones/fichas/poblacion/ficha vitales.pdf
16. Bustamante $\mathbf{P}$, Villa $A$, Lezana MA, Fernández R, Borja VH, Lona A, et al. El análisis de la desnutrición como causa múltiple de muerte. Salud Pública México. 1991;33:475-81.

17. Ortega A. Tablas de mortalidad. Centro Latinoamericano de Demografía (CELADE). Serie E, No. 1.004. San José de Costa Rica: Editorial CELADE; 1987.

18. González A. Conceptos y técnicas básicas del análisis demográfico. Primera edición. Bogotá, D.C.: Universidad Externado de Colombia; 2005.

19. Departamento Administrativo de Estadística Nacional. Colombia, tablas abreviadas de mortalidad nacionales y departamentales 1985-2020. Fecha de elaboración septiembre de 2007. Fecha de consulta: 24 de marzo de 2011. Disponible en: http://www.dane.gov.co/files/ investigaciones/poblacion/proyepobla06_20/8Tablasvida19 85_2020.pdf

20. Ministerio de la Protección Social, Departamento Nacional de Planeación, Ministerio de Agricultura y Desarrollo Social, Ministerio de Educación Nacional, Instituto Colombiano de Bienestar Familiar, Instituto Colombiano de Desarrollo Rural. Política Nacional de Seguridad Alimentaria y Nutricional PSAN. Bogotá: Dirección Nacional de Planeación; 2005. 